\title{
Gamma-Ray Spectral Characteristics of Thermal and Non-Thermal Emission from Three Black Holes
}

\author{
James C. Ling ${ }^{1 \star}$ and William A. Wheaton ${ }^{2}$ \\ 1 Jet Propulsion Laboratory 169-327, California Institute of Technology,4800 Oak Grove \\ Drive, Pasadena, CA 91109, USA \\ 2 Infrared Processing and Analysis Center, California Institute of Technology, 100-22, \\ Pasadena, CA 91125, USA
}

Received 2004 July 15; accepted 2004 month day

\begin{abstract}
Cygnus X-1 and the gamma-ray transients GRO J0422+32 and GRO J1719 - 24 displayed similar spectral properties when they underwent transitions between the high and low gamma-ray ( $30 \mathrm{keV}$ to few $\mathrm{MeV})$ intensity states. When these sources were in the high $\gamma$-ray intensity state $\left(\gamma_{2}\right.$, for Cygnus X-1), their spectra featured two components: a Comptonized shape below $200-300 \mathrm{keV}$ with a soft power-law tail (photon index $>3$ ) that extended to $\sim 1 \mathrm{MeV}$ or beyond. When the sources were in the low-intensity state $\left(\gamma_{0}\right.$, for Cygnus X-1), the Comptonized spectral shape below 200 keVtypically vanished and the entire spectrum from 30 $\mathrm{keV}$ to $\sim 1 \mathrm{MeV}$ can be characterized by a single power law with a relatively harder photon index $\sim 2-2.7$. Consequently the high- and low-intensity gammaray spectra intersect, generally in the $\sim 400 \mathrm{keV}-\sim 1 \mathrm{MeV}$ range, in contrast to the spectral pivoting seen previously at lower $(\sim 10 \mathrm{keV})$ energies. The presence of the power-law component in both the high- and low-intensity gamma-ray spectra strongly suggests that the non-thermal process is likely to be at work in both the high and the low-intensity situations. We have suggested a possible scenario (Ling \& Wheaton, 2003), by combining the ADAF model of Esin et al. (1998) with a separate jet region that produces the non-thermal gamma-ray emission, and which explains the state transitions. Such a scenario will be discussed in the context of the observational evidence, summarized above, from the database produced by EBOP, JPL's BATSE earth occultation analysis system.
\end{abstract}

Key words: gamma-rays observations - Black Holes - GRO J1719 - 24, GRO J0422+32 and Cygnus X-1

\section{INTRODUCTION}

auto-capitalized We have recently completed the processing of the full 9-year BATSE (Fishman et al. 1989) earth-occultation database, covering the period between

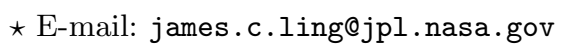

1991 and 2000, using the JPL Enhanced Occultation Package (EBOP, Ling et al.1996, 2000). This high-level database (levels 2 \& 3) consists of daily gamma-ray spectra with 14 energy channels between $35 \mathrm{keV}$ and 1.7 $\mathrm{MeV}$, and six broad-band flux histories with 1-day resolution for each of the 75 gamma-ray 


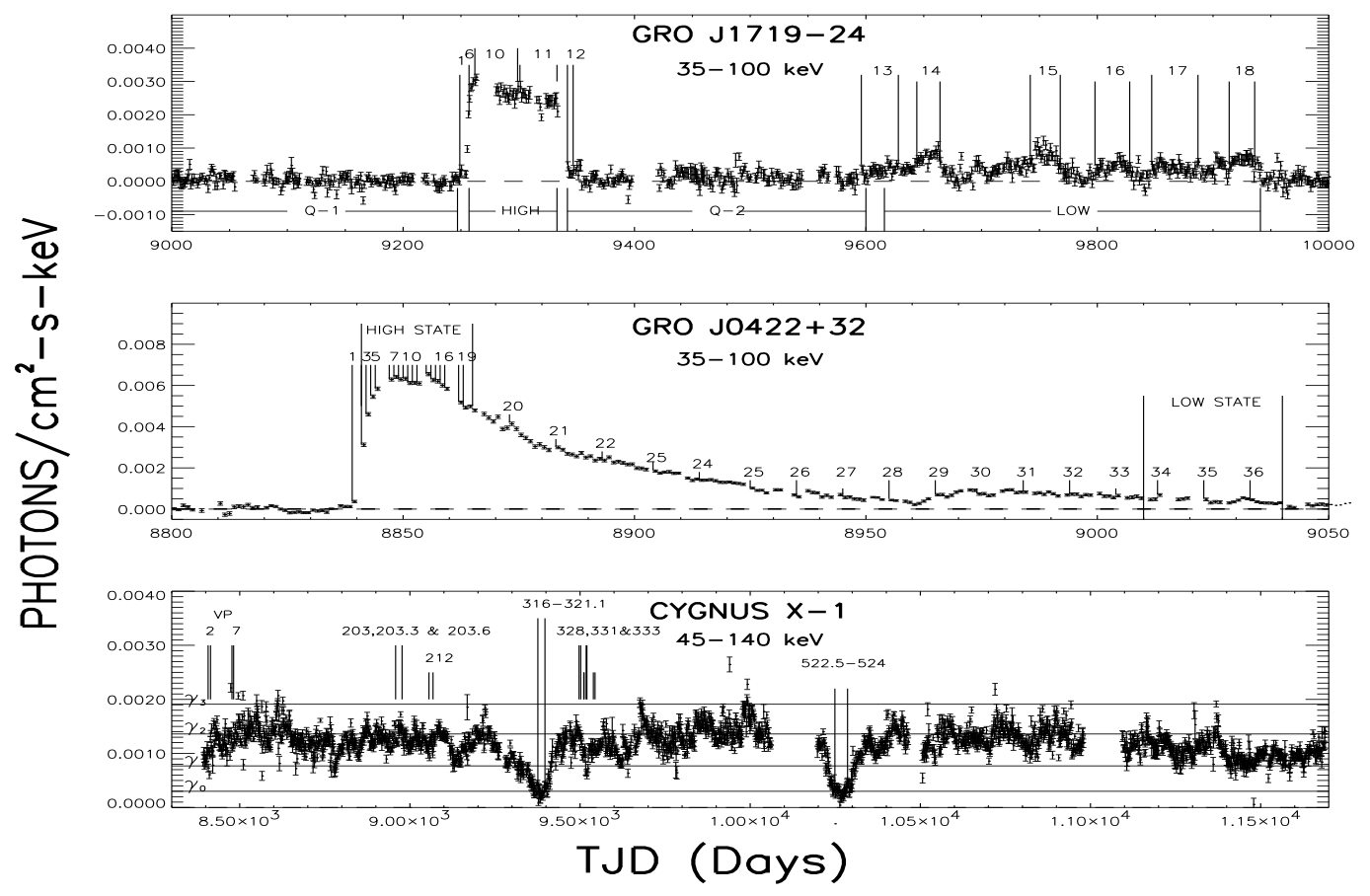

Fig. 1 Soft Gamma-Ray Flux Histories of GRO J1719 - 24, GRO J0422+32 and Cygnus X-1

sources, currently included in the EBOP source catalog. In this paper, we focus on the soft $\gamma$-ray emission of three black holes in our galaxy: GRO J1719 - 24 (Ling \& Wheaton 2004), GRO J0422+32 (Ling \& Wheaton 2003), and Cygnus X-1 (Ling et al. 1997; Zhang et al. 1997) observed by BATSE. All three sources underwent multiple gamma-ray flux transitions during this 9-year period between low and high-intensity gamma-ray states. During these transitions, all three sources displayed similar spectral properties and variability as described in the Abstract. Section 2 presents the long-term soft $\gamma$-ray flux histories and spectra of these sources observed by BATSE. Implications of these results in enhancing our understanding of these systems are discussed in Section 3.

\section{RESULTS}

Figure 1 shows the soft $\gamma$-ray flux histories of GRO J1719-24 (Ling \& Wheaton 2004), GRO
J0422+32 (Ling \& Wheaton 2003) and Cygnus X-1 covering the periods from 13 January 1993 to 10 October 1995 (TJD 9000-10000), 27 June 1992 to 23 April 1993 (TJD 8800-9100), and 24 May 1991 to 26 May 2000 (TJD 840011690, the full 9-year CGRO mission), respectively. During these periods, all three sources underwent multiple flux transitions.

GRO J1719 - 24 is a low-mass X-ray binary (LMXB) with a periodicity of 14.7 hours. The system consists of a $\sim 4.9$ solar mass compact object and a $\sim 1.6$ solar mass companion star (Masetti et al., 1996). The distance was estimated to be $\sim 2-2.8 \mathrm{kpc}$. The source was first discovered by BATSE (Harmon et al. 1993) and SIGMA (Ballet et al. 1993). Ling \& Wheaton (2004) showed that the hard X-ray flux rose suddenly from the quiescent level before 17 September 1993 (TJD 9247) to $\sim 1.5$ Crab 16 days later on 3 October (TJD 9263). It then slowly decreased in the next 70 days to 1 Crab level on 12 December (TJD 9333) (la- 

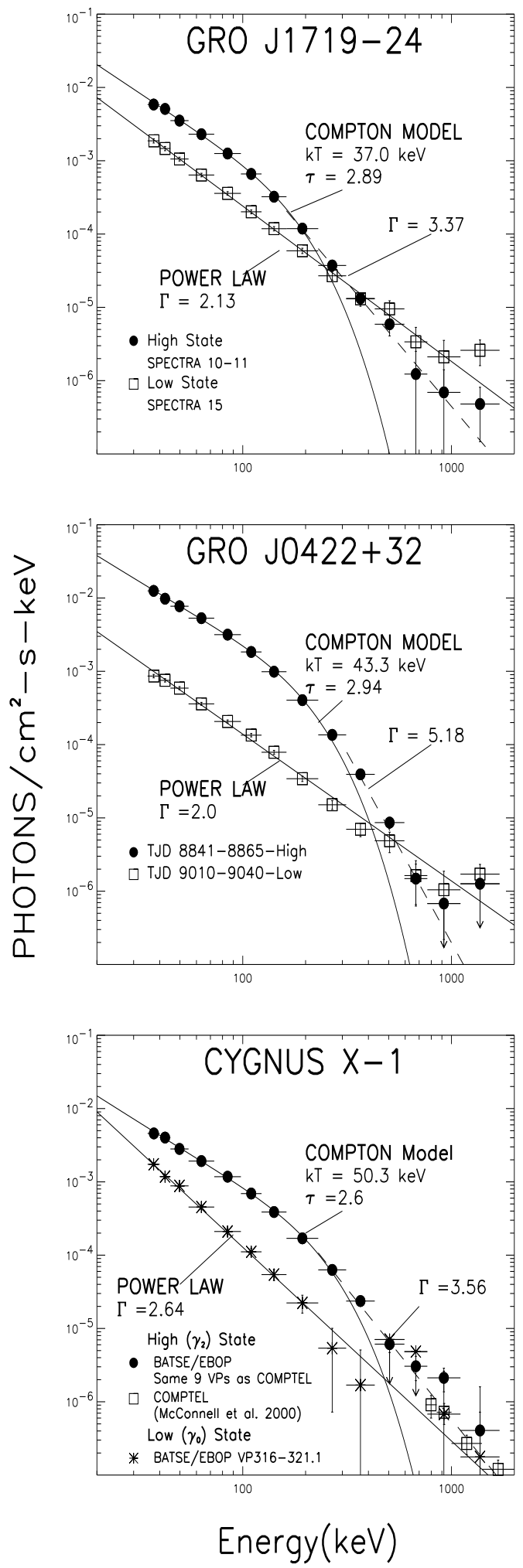

beled "10" and "11" in Figure 1 panel 1) before returning roughly to the pre-transition quiescent level where it remained until 5 September 1994 (TJD 9600). During the next 400 days between the fall of 1994 to the fall of 1995 (TJD 9600-10000), the source flared again five different times (marked as "14", "15", "16", "17", and "18" in Figure 1) to levels 3-7 times lower than the 1993 high-state level. Ling \& Wheaton (2004) showed that during the highintensity period between TJD 9262 and TJD 9333, BATSE observed high-energy gammaray fluxes up to $\sim 400-700 \mathrm{keV}$. The average fluxes in the $35-100 \mathrm{keV}, 100-200 \mathrm{keV}, 200-$ $300 \mathrm{keV} 300-400 \mathrm{keV}, 400-700 \mathrm{keV}$ and $700-$ $1000 \mathrm{keV}$ bands were measured to be $(2.57 \pm$ $0.02) \cdot 10^{-3}(\sim 1.2 \mathrm{Crab}),(3.23 \pm 0.05) \cdot 10^{-4}$ $(\sim 1.05 \mathrm{Crab}),(5.98 \pm 0.30) \cdot 10^{-5}(\sim 0.65$ Crab), (1.65 \pm 0.23$) \cdot 10^{-5}$ ( $\left.\sim 0.44 \mathrm{Crab}\right),(4.12$ $\pm 1.29) \cdot 10^{-6}(\sim 0.28 \mathrm{Crab})$, and $(1.00 \pm 0.89)$ $\cdot 10^{-6}$ ( $\sim 0.18$ Crab) photons $\mathrm{cm}^{-2} \mathrm{~s}^{-1}-\mathrm{keV}^{-1}$, respectively. During this 1000-day $\gamma$-ray flux excursion, Ling \& Wheaton (2004) showed that the source spectrum was best characterized by a power law during the first part of the rising phase from TJD 9249-9256. Then on TJD 9257, a day later, the spectrum changed suddenly from a power law to a Comptonized shape (Sunyaev \& Titarchuk 1980) below 200 $\mathrm{keV}$. It remained in this same Comptonized shape for the rest of the rising phase from TJD 9257 to TJD 9260 and when the source was in the high intensity state from TJD 9262 to TJD 9333. The spectrum then changed back to the power law for the rest of the 1000-day period when the source returned to the quiescent state and when the source made multiple transitions to the low-intensity states. Figure 2 (1st panel) shows that the average high-intensity state spectrum integrated over periods 10 \& 11 has two components: a soft power-law tail of photon index of $3.37_{-0.16}^{+0.19}$ above $200 \mathrm{keV}$, that extended to $\sim 500 \mathrm{keV}$, superposed on the low-energy Comptonized component below $200 \mathrm{keV}$. This is compared to a typical lowintensity power-law spectrum with a photon index of $2.13 \pm 0.05$ measured in period 15 . The two spectra intercept at $\sim 400 \mathrm{keV}$.

Fig. 2 Comparison of High vs. Low-Intensity Gamma-Ray State Spectra 
GRO J0422+32 is also a LMXB with a periodicity of 5.6 hours. The mass of the compact object is not well determined. Depending on the orbital inclination, a lower limit of $\geq 9 M_{\odot}$ is estimated. The distance of the source was estimated to be $\sim 2.4 \mathrm{kpc}$. The source was also discovered by BATSE in the summer of1992 (Paciesas et al. 1992) when its hard X-ray emission underwent a major outburst. Ling \& Wheaton (2003) showed that the $35-200 \mathrm{keV}$ flux rose sharply after the onset of the outburst on 5 August 1992 (TJD 8839, labeled " 1 "), and reached the first of two maxima during the peak of the outburst on 14 August (TJD 8848, labeled "7"). It then decreased slightly $(\sim 5 \%$ for $35-100 \mathrm{keV}$, and $\sim 6 \%$ for $100-200 \mathrm{keV}$ ) over the next few days before rising again to a second maximum on 21 August (TJD 8855, 13th tickmark). The declining phase of the event took $\sim 180$ days. The combined 35-200 keV flux reached half the peak level on $\sim$ TJD 8877, and a tenth of the peak on $\sim$ TJD 8935 , approximately 80 days later. It continued to decrease to a level about $4 \%$ of the peak value on TJD 8960 before slowly rising again to the so-called "secondary maximum" on TJD 8972 ( 120 days after the primary maximum on $\sim$ TJD 8852 ) at a level about $15 \%$ of the peak value. The $35-$ $200 \mathrm{keV}$ flux stayed within $20 \%$ of the "secondary maximum" for $\sim 20$ days before declining, and finally reached the "pre-outburst" quiescent level on TJD 9040. During this period, Ling \& Wheaton (2003) showed that the spectrum evolved from a power law on TJD 8839 (marked as "1") to a Comptonized shape below $200 \mathrm{keV}$ two days later on TJD 8841 (2nd tick mark) where it remained until $\sim$ TJD 8935 (marked as "26") before it changed back to a power law where it remained until the end of the period. The high-intensity spectrum, averaged over the period from TJD 8841-8865, shows two components: a Comptonized shape below $200 \mathrm{keV}$ followed by a soft power-law tail with photon index of $\sim 5.2$ that extends to $\sim 700 \mathrm{keV}$. The low-intensity spectrum, averaged over TJD $9010-9040$, is best described by a power law with photon index of $\sim 2$. The two spectra intersect at $\sim 600 \mathrm{keV}$.
Cygnus X-1, the best-known galactic black hole, is a high mass X-ray binary (HMXB) with a 5.6-day periodicity. It is well known for its bimodal X-ray $(1-10 \mathrm{keV})$ emission states. During an X-ray low to high-state transition, the hard X-ray flux $(>10 \mathrm{keV})$ was shown to be anti-correlated with the soft Xray $(<10 \mathrm{keV}$ ) flux (Ling et al. 1987; Zhang et al. 1997). Figure 1 (3rd panel) shows the $45-140 \mathrm{keV}$ lightcurve measured by BATSE covering the full nine years CGRO mission from 1991 to 2000. Included in the figure are the four flux levels $\left(\gamma_{0}, \gamma_{1}, \gamma_{2}, \& \gamma_{3}\right)$ defined by Ling et al. $(1987,1997)$. The source stayed at approximately the $\gamma_{2}$ state during most of this period. However, in two occasions, around TJD 9376 - 9397 (24 January-14 February 1994; labeled as VP 316 - 321.1) and TJD 10249 - 10286 (15 June-22 July 1996; VP 522.5 - 524), respectively, the source made a transition to the $\gamma_{0}$ state. The latter was clearly associated with the 1996 X-ray lowto-high state transition (Zhang et al. 1997), while the former was believed to be also (Ling et al 1997) although no contemporaneous Xray observations were available. Marked also in this figures are the nine CGRO Viewing Periods (VPs 2, 7, 203, 203.3, 203.6, 212, 328, 331 , \& 333) when contemporaneous $\gamma_{2}$ spectra measured by COMPTEL, OSSE and BATSEEBOP were compared (McConnell et al. 2000). Figure 2 (3rd panel) shows a comparison of the $\gamma_{2}$ spectrum measured by BATSE-EBOP and COMPTEL for the same period with the first $\gamma_{0}$ spectrum measured by BATSE in 1994. As previously reported in the literature (Ling et al. 1997; McConnell et al. 2000), the $\gamma_{2}$ spectrum shows two components, a Comptonized shape below $300 \mathrm{keV}$ followed by a power law from $300 \mathrm{keV}$ to $\sim 1 \mathrm{MeV}$. The $\gamma_{0}$ spectrum (Ling et al. 1997; Phlipset al. 1996), on the other hand, shows a single power law with photon index of $\sim 2.6-2.7$. The two spectra intersect at $\sim 1 \mathrm{MeV}$. McConnell et al. (2002) reported that the same $\gamma_{2}$ spectrum intersects the 2 nd $\gamma_{0}$ spectrum measured in 1996 at $\sim 1$ $\mathrm{MeV}$ also. 

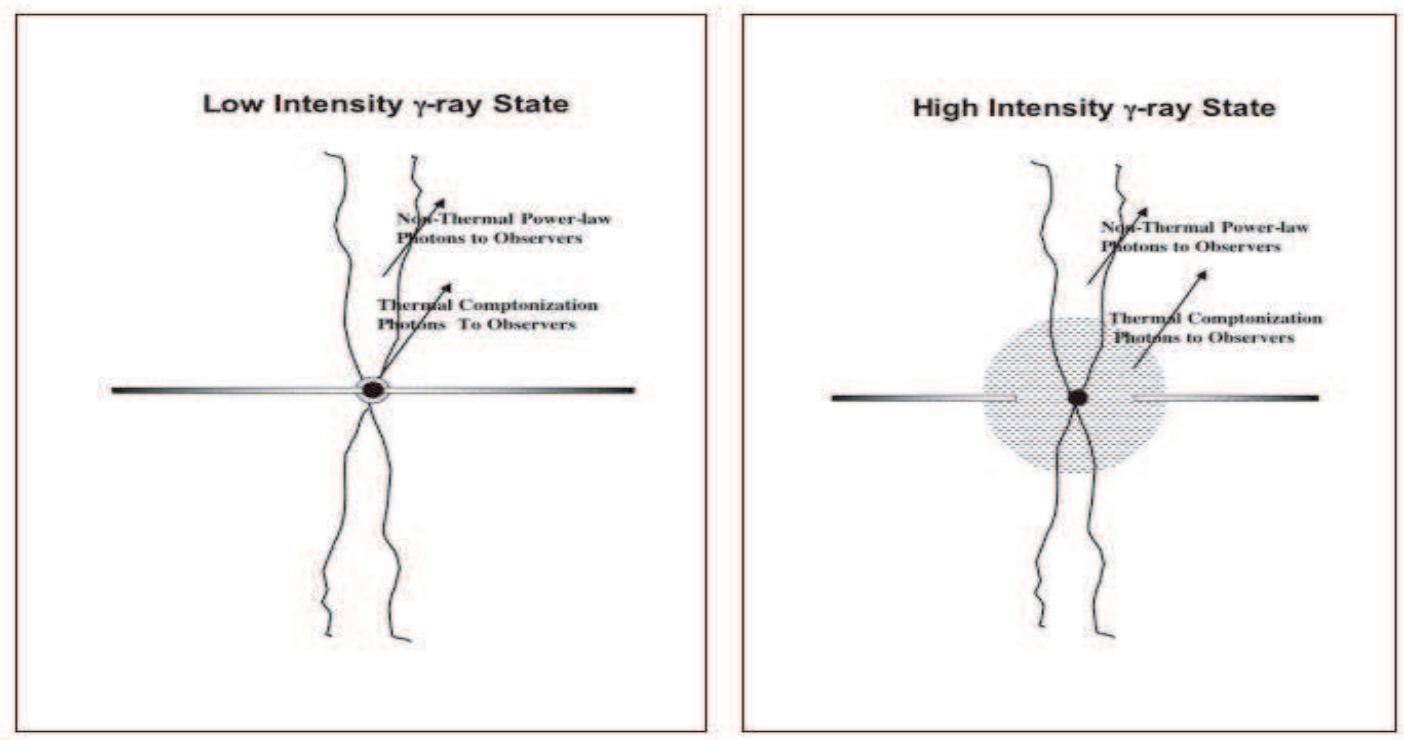

Fig. 3 System Configurations for High and Low Gamma-Ray Intensity States

\section{DISCUSSION}

GRO J1719-24 (Ling \& Wheaton 2004), GRO J0422+32 (Ling \& Wheaton 2003) and Cygnus X-1 (Philips et al, 1996; Ling et al. 1997; McConnell et al. 2000, 2002) are three galactic black holes that displayed similar gamma-ray spectral characteristics when undergoing transitions between the high and low $\gamma$-ray intensity states. The low-intensity states were described by a power law with spectral index of $\sim 2-2.7$. The high-intensity states were well fit by a two-component spectrum: a Comptonized component below $\sim 200 \mathrm{keV}$ with electron temperature $k T_{e} \sim 37-50 \mathrm{keV}$ and optical depth $\tau \sim 2.6-3$, and a soft power-law tail with photon index of $>3.1$ above $200 \mathrm{keV}$. These two distinct gamma-ray state spectra are clearly different than the simple power law spectrum shown in GRO J1655-40, and a broken power law shown in GRS1915+105 (Case et al. 2004), two well-known microquasars in our galaxy, suggesting that there are perhaps two different classes of gamma-ray emitting $\mathrm{BH}$ candidates in our galaxy.
The persistent power-law emission seen in both high and low-intensity spectra of these sources suggests that non-thermal emission may be at work in both situation. Nonthermal emission may be associated with jets which Meier (2001) claimed is a natural consequence of accretion flows onto rotating black holes. More recently, Meier's (2004) model of Magnetically-Dominated Accretion Flows (MDAF) further develops the idea and integrates accretion and jet production as an extension of the ADAF (Esin et al. 1998) and Shakura \& Sunyaev (1976) disk models. Non thermal emission may also be associated with Compton scattering off relativistic electrons of free infalling matter onto the black hole in the converging flow region near the event horizon (Chakrabarti \& Titarchuk 1995; Turolla et al. 2002). Over the years. there have been also attempts to develop a hybrid thermal/non-thermal Comptonization model (Coppi 1998; Gierlinski et al.1999) to explain the two-component high-intensity gamma-ray spectra observed in BH binary systems such as GRO J1719 - 24, GRO J0422+32 and 
Cygnus X-1, but no attempts have been made to explain how the spectrum evolved into a power law in the low-intensity scenario. Ling \& Wheaton (2003) suggested a possible system scenario for explaining both the high and low-intensity spectra (see Figure 3). It is based on the ADAF model of Esin et al (1998) along with the source geometry envisioned by Poutanen \& Coppi (1998) and others. For the high-intensity scenario (Figure 3 right panel), the system consists of a hot inner corona, a cooler outer thin disk, and a separate region that produces the power-law $\gamma$-ray emission. We hereby refer to the latter the "Non Thermal Emission Region (NTER)". NTER may include a jet (see Figure 3) and possibly also the converging flow region discussed above. Under such condition, the transition radius of the disk is $\sim 100$ Schwarzschild radii from the black hole. Electrons in the hot corona up-scattered the low-energy photons produced both inside the corona as well as from the outer disk to form the Comptonized component that dominates the spectrum in the 35-200 keV range. These same electrons also down-scattered the high energy photons $(>10$ $\mathrm{MeV}$ ) produced in NTER resulted in forming a softer power-law component observed in the $300 \mathrm{keV}$ to $1 \mathrm{MeV}$ range. Under the lowintensity scenario that could be triggered by a significant increase of the accretion rate, a large quantity of soft photon flux was produced in the disk that cooled and quenched the hot corona and movedthe transition radius inward to a distance very close to the horizon. Under this condition, the Comptonized component below $200 \mathrm{keV}$ disappeared and the entire 35-1000 keV spectrum is dominated by the unperturbed power-law emission produced in NTER.

Acknowledgements We wish to thank Gerald Fishman and his BATSE team for their support of the JPL EBOP development throughout the years, and Michael Cherry and Gary Case for their comments on this paper. The work described in this paper was carried out at the Jet Propulsion Laboratory under the contract with the National Aeronautics and Space Administration.

\section{References}

Ballet, J., Denis, M., Gilfanov, M., Sunyaev, R. 1993, IAU Circ. 5874

Case, G. L. et al., 2004, these Proceedings

Chakrabarfti, S. K., \& Titarchuk, L.. 1995, ApJ, 455, 623 .

Coppi, P. S. , 1998, "The Physics of Hybrid Thermal/Non-thermal Plasmas" in High energy Porcesses in Accreting Black Holes, eds. J Poutanen and R. Svensson, ASP Conf. Series, Vol. 161, p. 375 (astro-ph/9903158)

Esin, A. A. et al., 1998, ApJ, 505, 854.

Fishman, G. J. et al., 1989, in Proc. Gamma Ray Observatory Science Workshop, ed. W. Johnson (Greenbelt:GSFC), 2.

Gierlinski, M. et al., 1999, MNRAS, 309, 496.

Harmon, B. A. et al., 1993a, IAU Circ. 5874

Harmon, B. A. et al., 1993b, IAU Circ. 5900

Ling, J. C., and Wheaton, W. A., 2003, ApJ, 384, 399.

Ling, J. C., and Wheaton, W. A., 2004, submitted to ApJ.

Ling, J. C., et al., 1996, A\&AS, 120, 667.

Ling, J. C., et al. 1987, ApJL, 321, L117.

Ling, J. C., et al. 1997, ApJ, 484, 375

Ling, J. C., et al. 2000, ApJS, 127, 79

Masetti, N. et al., 1996, A\&A, 314, 123.4

McConnell, M. L., et al. 2000, ApJ, 543, 928.

McConnell, M. L., et al. 2002, ApJ, 572,: 984.

Meier, D. L., 2001, ApJ, 548, L9.

Meier, D. L., 2004, private communication.

Paciesas, W. S., et al., 1992, IAU Circ. 5580.

Phlips, B., et al., 1996, ApJ, 465, 907.

Poutanen, J. \& Coppi, P. S. 1998, Physica Scripta, T77, 57 (astro-ph/9711316).

Shakura, N.I. \& Sunyaev, R. A. 1976, MNRAS, 175,613 .

Sunyaev , R. A., \& Titarchuk, L. G., 1980, A\&A, $86,121$.

Turolla, R., Zane, S., \& Titarchuk, L., 2002, ApJ, 576,349 .

Zhang, S. N. et al., 1997, ApJL, 477, L95.

This paper was prepared with the ChJAA LTEX macro v1.0. 